\title{
CORRECTIONS
}

\section{Obituary: Michael Lasserson}

The obituary for Michael Lasserson gave the wrong year of birth (BMJ 2011;343:d7757, doi:10.1136/bmj.d7757). Lasserson

was born in 1942 and not 1938. The online version has been corrected. 